\title{
Traduire la recherche en pratiques: vers un acte de transformation et d'engagement
}

\section{Marina Schwimmer}

En s'inspirant des travaux issus de la philosophie de l'éducation et en adoptant une perspective empruntée à la philosophie du langage, cet essai tente de se distancier des conceptions habituellement retenues pour discuter la traduction de la recherche en pratiques en éducation. Après avoir brièvement présenté un modèle de la traduction comme acte de transformation et d'engagement, il examine la fertilité de ce modèle au regard des travaux de Paul Standish sur la justice sociale. Puis, à partir de ce modèle, il examine un débat important des sciences de l'éducation: la question du rapport entre la recherche et la pratique. Enfin, il présente deux questions soulevées par ce modèle, d'abord la question des critères qui permettent de juger les qualités et la pertinence de la recherche, puis la question des interfaces de traduction. Ce faisant, il dégage quelques implications pour l'enseignement, la recherche en éducation ainsi que la formation des maîtres.

\section{Introduction}

La question de la traduction se pose normalement dans le but de réfléchir aux règles et processus qui assurent la transposition des textes d'un code linguistique à un autre. Cependant, il s'agit également d'une image fertile pour penser le monde actuel, c'est-à-dire un monde complexe et fondamentalement pluriel. Dans le champ de la philosophie de l'éducation anglo-saxonne, la notion de traduction connaît une effervescence intéressante. Elle est utilisée pour réfléchir à différents objets, notamment le travail de l'enseignant comme acte de traduction (Higgins \& Burbules, 2011; Dobson, 2012), la traduction comme projet de développement personnel ou Bildung (Saito, 2009), la traduction en contexte de mondialisation (Berghdal, 2009; Harris, 2009) et la traduction comme méthode philosophique (Ruitenberg, 2009; Standish, 2011). Le présent numéro thématique s'intéresse notamment à la traduction comme processus assurant le passage de la recherche en éducation vers les milieux de la pratique. Cet essai propose une réflexion sur ce que les avancées produites dans le champ de la philosophie de 
l'éducation peuvent apporter pour éclairer ce problème particulier des sciences de l'éducation et pour faire surgir de nouvelles questions.

L'essai est structuré autour de quatre axes. D'abord, il esquisse un modèle de la traduction comme acte performatif de transformation. Puis il examine la pertinence de ce modèle au regard des travaux issus du champ de la philosophie de l'éducation, notamment ceux de Paul Standish (2011) sur la justice scolaire. Ensuite, il centre l'analyse sur les traductions d'un objet de débat important actuellement dans les contextes éducatifs internationaux: le rapport entre la recherche et la pratique. Enfin, il réfléchit à deux enjeux soulevés par le modèle de traduction présenté: d'abord la question des critères qui permettent de juger les qualités et la pertinence de la recherche, puis la question des interfaces qui séparent/unissent la recherche et la pratique dans ce cadre. Ce faisant, il dégage quelques implications pour l'enseignement, la recherche en éducation ainsi que pour la formation des maitres.

\section{La traduction comme acte de transformation et d'engagement}

Dans son célèbre essai sur la tâche du traducteur, Walter Benjamin (2000) affirme que la traduction n'est pas la transmission d'un texte original, mais sa réalisation; qu'elle constitue une transformation et un renouvellement d'une chose vivante et crée un nouveau système de signes qui n'imite pas l'original, mais le complémente. C'est dire que la traduction n'est pas seulement un acte de restitution du sens d'un texte mais qu'elle participe inévitablement à sa transformation. Comme le note Michaël Oustinoff (2003), la vision dominante chez les traductologues est de considérer que la différence produite par la traduction est nécessairement une imperfection ou une "défectivité» et son défaut majeur est sa secondarité (p. 57). Mais selon une certaine tradition de pensée issue de la philosophie du langage, la traduction est à elle seule, acte d'écriture. C'est Henri Meschonnic (1973) qui a élaboré cette théorie de la traduction, en s'inspirant de Humbolt, Heidegger, Benjamin. Pour lui, l'idée de restituer le sens d'un texte dualise l'original et la traduction et réduit nécessairement le sens d'un texte toujours plurivocal à la monosémie. Il défend que la traduction est plutôt un travail dans la langue, un décentrement: elle cherche elle aussi à être une œuvre.

À ce titre, il peut être utile de rappeler la distinction effectuée par John Langshaw Austin (1975) entre les actes de discours constatifs et les actes de discours performatifs. Les premiers sont des énoncés narratifs qui décrivent le monde et ont valeur de vérité, par exemple lorsque le professeur explique une théorie ou lorsqu'il présente des typologies scientifiques. Les seconds sont dits performatifs car ils accomplissent une action par leur énonciation. Lorsque le juge rend sa sentence ou que le professeur donne une note à un élève, il performe son énoncé en l'énonçant. Mais cette opposition est contestable. Selon Jacques 
Derrida (1972b) par exemple, on ne peut distinguer d'une part des énoncés constatifs et, d'autre part, des énoncés performatifs, comme s'il s'agissait de deux fonctions séparées. Plutôt, chaque énoncé comporte ces deux dimensions. En décrivant une réalité, on participe toujours activement à la réalisation de son sens.

Dans l'essai La pharmacie de Platon, Derrida (1972) déconstruit le concept de «Pharmakon" présent dans Le Phèdre. En grec classique, "Pharmakon" possède plusieurs significations paradoxales. Il s'agit à la fois d'un poison, d'un remède, d'un philtre, d'une recette, d'une drogue, etc. Aucune traduction ne pourrait rendre compte de la polysémie interne qui caractérise le texte original. Tenter de traduire "Pharmakon», c'est entrevoir qu'il y a au cœur du langage une indécidabilité et la nécessité d'assumer soi-même le sens à assigner. On critique souvent le postmodernisme de Derrida, mais ce dernier n'est pas un anarchiste, un nihiliste ou un antimoderne. Au contraire, cette traductibilité inhérente aux signes constitue pour lui une réalité fondamentale sur la nature des signes. Et le fait que le signe soit ouvert à l'assignation du sens implique un espace qui appelle à être investi. Derrida (1979) affirme: «Seule l'absence pure - non pas l'absence de ceci ou de cela, - mais l'absence de tout où s'annonce toute présence, peut inspirer, autrement dit travailler, puis faire travailler.» (p.17) C'est-à-dire que cette ouverture du signe appelle à se mettre au travail, à s'investir, à s'engager.

Plus récemment, le philosophe du droit François Ost (2009) a utilisé la notion de traduction pour réfléchir aux problèmes que pose la transposition des lois d'une nation à l'autre ou l'application des lois internationales dans différentes nations. De façon assez provocante, il affirme que la traduction est une érotique car elle laisse toujours à désirer. Elle laisse à désirer car elle est toujours imparfaite. Mais aussi, elle laisse à désirer car elle renvoie à un espace de désir qui attend l'assouvissement. C'est-à-dire que le texte à traduire n'est jamais saturé de sens, son sens est toujours encore à réaliser, ouvert à l'appropriation, il offre une opportunité de transformation et donc, d'engagement.

\section{Traduction du concept de justice scolaire}

Les tentatives d'utiliser la traduction comme modèle d'analyse ont également occupé quelques philosophes de l'éducation. Claudia Ruitenberg (2009) réfléchit notamment à la manière dont la traduction de certaines expressions donne lieu à de nouvelles significations inter-linguistiques et inter-discursives. En défamiliarisant le langage, la traduction ouvre de nouveaux espaces de compréhension. Par exemple, le verbe "to know» en anglais peut être traduit en français par «savoir» ou par "connaître», deux activités qui soulèvent des problèmes différents. On pourrait discuter indéfiniment des nuances entre les deux termes, mais l'important ici est de retenir qu'en traduisant un concept, on le place dans un contexte moins familier qui fournit la distance nécessaire pour développer de nouvelles perspectives. Dans la même veine, Standish (2011) propose une 
analyse très intéressante de la traduction du concept de justice sociale. Il soulève le fait que pour un chinois ou un japonais, par exemple, le concept sera largement teinté par les acceptions spécifiques de ce qu'est la justice ou la société dans sa culture. Cette plurivocalité pourrait cependant passer complètement inaperçue pour un anglophone unilingue.

La majorité des écrits en éducation qui traitent des questions de justice est produite dans la foulée des travaux de John Rawls ou dans le champ de la pédagogie critique. Dans un régime de sens rawlsien, la justice scolaire se traduirait par la distribution équitable des biens éducatifs. Pour les pédagogues critiques, elle se traduirait par les vertus de reconnaissance de l'autre, de valorisation de la différence, etc. Mais comme le soulève Standish (2011), ces régimes de sens passent à côté de certains aspects cruciaux de la réalité éducative qui ont trait à la substance même de l'enseignement et de l'apprentissage, c'està-dire la transformation de l'être humain (le développement de l'intelligence, de la créativité, de la sensibilité, etc.). Ce qui pose problème ici, ce n'est pas la tradition rawlsienne ou celle de la pédagogie critique, mais leur dominance discursive concernant la justice en éducation: il ne faudrait pas que ces traditions préviennent indirectement la possibilité de penser, traduire la justice autrement.

Par exemple, la notion de conversation développée par Stanley Cavell (2004), qui est indirectement influencée par la tradition confucéenne, permet de tenir compte de l'aspect transformateur de l'éducation, car elle accentue le processus par lequel il devient possible de découvrir ce que mon (ou notre) projet de justice pourrait être et non d'accomplir ce qu'il devrait être déjà a priori. La notion de conversation met l'accent sur l'état actuel et réel des interactions, qu'elles soient coopératives ou antagonistes. Les principes de la justice deviennent questions de débats, sujets de conversation, lieux d'apprentissage et de développement. Les vertus valorisées ne sont plus seulement l'équité ou la reconnaissance, mais aussi l'écoute, la conscience de l'altérité, la volonté de changer. Précisons qu'il ne s'agit pas de choisir entre ces différentes conceptions de la justice, mais seulement de s'assurer que la prédominance de l'une ne prévienne pas la possibilité de penser les autres (Cavell dans Standish, 2011).

Si la traduction permet de sortir de nos régimes de sens habituels, c'est parce que le travail dans la langue permet de prendre la distance nécessaire pour les déstabiliser. Il faut préciser que cette opération n'est pas seulement une réalité interlinguistique, elle se performe aussi à l'intérieur d'une seule langue. Cela est particulièrement visible lorsque nous tentons, par exemple, de rapporter les propos de quelqu'un d'autre dans la même langue: parce qu'il a un autre tempérament et une autre sensibilité, ses mots sonnent différemment si nous les employons, alors nous devons les reformuler dans notre langage pour les respecter (Oustinoff, 2003). ${ }^{1}$ Ce travail dans le langage est aussi un travail de traduction. C'est d'ailleurs parce que toute langue est toujours en train de se traduire elle-même, qu'elle peut évoluer, se transformer et créer de nouvelles significations ${ }^{2}$. Je propose donc ici de réfléchir à une nouvelle traduction possible 
du rapport entre la recherche et la pratique et donc de subvertir en quelque sorte le sens de la notion même de traduction telle qu'on l'entend habituellement lorsqu'on parle de «traduire la recherche en pratiques».

\section{Traduire la recherche en pratiques}

De façon générale, on peut distinguer trois manières de concevoir le rapport entre la recherche et la pratique (voir par exemple Hofstetter, Schneuwly \& Lussi, 2005). La première: la recherche fondamentale peut informer les pratiques. La deuxième: la recherche doit être socialement pertinente et se mettre au service des pratiques. Dans ce cas, la recherche permet de développer des savoirs immédiatement pertinents et mobilisables pour l'enseignement. Et la troisième: les deux mondes sont incommensurables. Dans ce cas, il y aurait une incompatibilité structurelle qui rend la recherche relativement intraduisible. Ces trois perspectives induisent des modèles de traduction de la recherche en pratiques différents.

La première conception induit un modèle de traduction comme éclairage. La recherche peut fournir des idées, des exemples, pour informer ou inspirer des pratiques. Mais en réalité il est difficile d'identifier concrètement quelles sont ici les opérations de traductions en jeu. La recherche vise surtout à mettre des dimensions éducatives en lumière et n'est pas traduite à proprement parler par une instance intermédiaire. Ce sont les praticiens qui s'approprient et interprètent le sens de la recherche qui est initialement produite dans une visée d'approfondissement de la connaissance. La traduction est alors à la fois un phénomène de mise à disposition aléatoire de la part des chercheurs et d'interprétation active de la part des praticiens. J'y reviendrai plus loin.

Cependant, dans le contexte des mouvements internationaux de professionnalisation de l'enseignement actuels, c'est la deuxième perspective qui domine. L’idée est qu'il existe des bonnes pratiques («Best Practices») et que le rôle de la recherche éducative est principalement de les identifier et de les diffuser. Les organisations internationales de recherche comme le CERI et l'OCDE affichent une nette sympathie pour ces développements. Au Québec, cette tendance est encore timide, mais elle commence à avoir ses défenseurs, notamment Steve Bissonnette, Mario Richard et Clermont Gauthier (2005). Ainsi qu'en témoignent le rapport du Conseil supérieur de l'éducation sur le dialogue entre la recherche et la pratique (CSÉ, 2006) et le document ministériel sur la gestion des connaissances en éducation (MELS, 2009), la nécessité d'améliorer les pratiques éducatives grâce à la recherche scientifique ou académique est au centre des préoccupations actuelles. On attend de plus en plus que les chercheurs rendent leurs recherches plus pertinentes et accessibles pour les praticiens, et que les enseignants utilisent la recherche de façon active dans leurs pratiques dans le but de réduire le supposé fossé qui sépare la recherche et la pratique. 
Dans ce contexte, "traduire la recherche en pratiques», c'est avant tout adapter la recherche dans le but d'assurer un bon transfert des connaissances vers les utilisateurs potentiels. Comme l'indique le document gouvernemental, cette étape consiste à transformer les connaissances générées par les chercheurs pour en faciliter l'accessibilité et l'utilisation par les praticiens. (MELS, 2009) ${ }^{3}$

Jbilou (2010) précise que le bon transfert des connaissances repose sur la capacité rendre les résultats de recherche accessibles aux praticiens, tant d'un point de vue cognitif (simplifier la présentation des résultats de recherche) que pratique (expliciter les implications pratiques des résultats de recherche). Mais cette façon de concevoir la traduction pose des problèmes fondamentaux. Elle repose essentiellement sur un modèle instrumental de la recherche selon lequel il est possible et souhaitable que le savoir soit objectivé et l'être humain suffisamment rationnel pour assurer la meilleure performativité du système. La majorité des chercheurs s'entend, au moins depuis Le praticien réflexif de Donald Schön (1994), pour dire qu'il y a dans l'enseignement une importante part de savoir tacite qui procède du jugement pratique et non de la connaissance propositionnelle ou procédurale. Mais les réformes ne cessent quand même de valoriser un modèle instrumental du savoir pour l'enseignement en imposant des modèles pédagogiques, des méthodes d'enseignement ou des pratiques d'évaluation à appliquer, comme s'il était possible de produire un savoir entièrement détaché de la personne sensée savoir. Les universités continuent également à promouvoir ce modèle en offrant des formations initiales devant supposément être appliquées subséquemment dans la pratique.

Une autre manière courante de comprendre la traduction de la recherche en pratiques renvoie à la troisième perspective présentée plus haut, c'est-à-dire en référence à la traduction entre deux langages: le langage académique des chercheurs et le langage professionnel des enseignants. En effet, le problème du fossé entre la recherche et la pratique est souvent présenté comme un fossé culturel entre le monde des chercheurs et celui des praticiens. Par exemple, David Labaree (2003) parle d'une certaine rupture entre les deux cultures:

from a normative to an analytical way of thinking, from a personal to an intellectual relationship with educational phenomena, from a particular to a universal perspective, and from a experimental to a theoretical disposition. (p. 13)

Allant dans le même sens, Martyn Hammersley et Roger Gomm (dans Hammersley, 2002) soutiennent que la limite principale du modèle «applicationniste» de la recherche est de supposer qu'il existe un monde unique dans lequel chercheurs et praticiens pourraient communiquer de façon transparente. Selon eux, les chercheurs et les praticiens ont des langages et des horizons différents, ce qui est d'ailleurs souhaitable, et sont donc inévitablement confrontés à la nécessité de la traduction. Comme il est impossible de savoir ce qui sera retenu comme pertinent par tel ou tel praticien en fonction de sa situation particulière, on ne peut jamais savoir exactement quelles seront les traductions, et 
donc quelles seront les retombées de la recherche. Ce qui est intéressant avec cette analyse est qu'en admettant que les divergences culturelles donnent lieu à des traductions imprévisibles (c'est le propre de la traduction), elle conclut qu'il faudrait donc reconnaitre que nous surestimons le rôle que peut jouer la recherche pour améliorer directement les pratiques. Cette façon de présenter le problème touche des points très importants, cependant elle paraît centrer le problème de la traduction autour d'un problème essentiellement culturel. Mais si l'on accepte de se décentrer des acceptions habituelles de la traduction et de prendre le point de vue de la philosophie du langage, la traduction n'est pas seulement un problème de transposition entre des mondes ou des cultures différentes, il s'agit plus profondément encore d'un problème lié à la nature même du langage: le langage fonctionne sur le mode de la traduction perpétuelle.

J'aimerais pousser plus loin cette façon moins courante de concevoir la traduction de la recherche en pratiques. Pour aborder cela, je pense que la notion de "dissémination» aujourd'hui communément utilisée pour parler du phénomène de diffusion de la recherche universitaire est utile. Comme l'indique Derrida (1972), la vision commune est ancrée dans un schéma selon lequel le sujet accède à la connaissance par la réception des idées produites par l'histoire et donc "dans la servilité de l'écoute». Elle exclut le travail actif et créateur de celui qui prend en charge le sens de l'histoire et cherche à la rendre fertile. Pour se décentrer de cette acception, il est nécessaire de retravailler un peu la langue en allant explorer les autres significations qu'elle renferme. Sans aller chercher très loin, on retrouve dans la notion de dissémination l'idée de dispersion des semences. Et dans ce registre de sens-là, il est évident que malgré les tentatives de contrôler le processus de semence, on ne peut savoir quelles graines seront fertiles ni où le vent, ou les oiseaux, les mèneront. Derrida (1972) exprime ainsi:

La dissémination marque une multiplicité irréductible et générative. Le supplément et la turbulence d'un certain manque fracturent la limite du texte, interdisent sa formalisation exhaustive et clôturante ou du moins la taxinomie structurante de ses thèmes, de son signifié, de son vouloir dire. (p. 62)

En ce sens, «traduire la recherche en pratiques», c'est actualiser un potentiel qu'on ne peut ja-mais avoir tout à fait planifié. Comme la traduction littéraire est un travail dans la langue, la traduction de la recherche est un travail dans la pratique. Comme le note Françoise Lantheaume (2007), ce travail procède par tâtonnements, par une intelligence rusée, une ingéniosité qui se développe dans la nécessité du moment présent et qui, sans être entièrement prise en charge par la raison, sans être entièrement calculée ou maîtrisée, demeure source effective de savoir. Ce travail exige donc de se risquer à tout instant malgré l'incertitude et de s'engager devant l'indécidable. En fait, comprise ainsi, la traduction ne peut plus être considérée comme le travail «d'agents de liaison» externes, mais bien comme celui des praticiens eux-mêmes. La dualité tra-ditionnelle entre recherche et pratique est alors implicitement abolie. 
Cette troisième façon d'appréhender la notion de traduction induit une nouvelle conception du rapport entre la recherche et les pratiques et semble faire surgir de nouveaux questionnements. Si la traduction est un acte de transformation, de tâtonnements et d'engagement, alors la recherche ne peut être entièrement objectivée, puis appliquée. Dans ce contexte, on peut se demander quel est le rôle de la recherche par rapport à la pratique. Plus spécifiquement, on peut se demander quels sont les critères qui permettent de juger la qualité et la pertinence d'une recherche si ce n'est son applicabilité directe. On peut aussi se demander quel type d'interfaces de traduction valoriser dans une économie du savoir qui n'est pas fondée sur l'application des savoirs homolo-gués. J'essaierai de répondre à ces deux questions.

\section{Comment juger la qualité et la pertinence de La recherche?}

Dans un premier lieu, si l'appropriation de la recherche a toujours quelque chose d'imprévisible, alors comment juger si une recherche est bonne ou pertinente? Selon le modèle actuel de la professionnalisation de l'enseignement, les recherches fournissent des résultats clairs, explicites, mesurables qui peuvent être mobilisés par le personnel scolaire dans la classe ou l'école. Ce sont habituellement ces critères d'objectivité et d'applicabilité qui permettent de juger de la qualité et de la pertinence de la recherche. Ces critères entrent dans une économie où l'on cherche à contrôler la manière dont la recherche sera traduite en pratiques. Or, nombre d'expériences de réformes ratées, de rapports de recherche oubliée, de formations inutiles, montre bien comment tenter de contrôler entièrement l'appropriation de la recherche est une tâche téméraire. Dans ce cas, ne serait-il pas préférable de travailler avec cette donnée plutôt que de continuer à la nier? C'est ce que je propose ici en accentuant l'importance de valoriser aussi d'autres normes de qualité et de pertinence.

Les conceptions traditionnelles de la traduction de la recherche en pratiques entrent dans une économie de l'échange et de la satisfaction, c'est-à-dire une économie fermée qui totalise le champ concerné. Dans ce type d'économie, un enseignant qui rencontre un problème dans sa salle de classe consulte la recherche académique pour trouver des solutions précises à son problème. Le directeur d'établissement organise des séances de développement professionnel pour mettre son personnel au fait des nouvelles pratiques en vogue. Le savoir est rentabilisé: il n'y a ni excès ni perte. Dans la même veine, un étudiant en formation des maîtres ou en formation continu remplit un contrat: il reçoit un plan de cours, prend connaissance des exigences et des attentes et les remplit. On encourage les étudiants à concentrer leurs efforts sur la manière la plus efficace remplir ces exigences et ces attentes. $\mathrm{Ni}$ excès, ni pertes. Or, ce type d'économie 
ne laisse-t-il pas de côté le meilleur de la recherche, de l'enseignement et de l'apprentissage?

En référence à l'éducation universitaire, Paul Standish (2005) propose de penser une économie de l'excès: celle-ci se caractérise par un engagement qui s'approfondit et s'intensifie au fur et à mesure qu'on y répond. Dans une telle économie, la recherche n'est pas seulement un outil d'efficacité, elle permet d'accéder à cette profondeur et cette intensité. Qui n'a jamais lu un texte, assisté à une conférence ou un cours qui l'a soudainement transporté, qui l'a rempli d'une énergie nouvelle, d'une urgence inattendue de se mettre au travail? Aussi, en tant que chercheurs, nous connaissons aussi ce sentiment d'enthousiasme et d'excitation qui naît dans l'écriture quand soudainement nous avons l'impression de toucher quelque chose d'important ou quand nous trouvons les bons mots pour exprimer une idée. Il y a dans ce moment d'exaltation quelque chose qui nous dépasse, comme si on répondait à un appel. Et plus nous approfondissons notre pensée, notre recherche, plus le désir d'y répondre s'intensifie. Dans ce cadre, une bonne recherche n'est pas nécessairement une recherche précise, réplicable, objective (bien que ce type de recherche puissent aussi être tout à fait valable dans certains contextes), mais est peut-être aussi une recherche qui inspire l'enseignant et lui insuffle le désir de se mettre au travail. La recherche ne peut donc être qu'une recherche où le praticien est lui-même engagé d'une façon ou d'une autre.

Les enseignants en formation ou débutants espèrent souvent des recherches leur présentant des connaissances toutes faites, déjà "traduites», pour réduire l'incertitude de leur travail, leur vulnérabilité devant l'imprévisible. Mais céder à ces attentes participe à nier cette activité, cette vitalité. Le travail du traducteur n'est pas d'appliquer mécaniquement des transformations, mais d'être plongé corps et âme dans l'action. En ce sens, le traducteur doit être dans un rapport intime avec le savoir qu'il développe ou incarne et ce savoir ne devrait jamais être trop figé, univoque, directif. Et si ce savoir provient de textes produits par d'autres et non de l'expérience directe, ces textes devraient être traversés par la pluralité et la complexité, appeler le questionnement et le doute, et engager la participation et l'enthousiasme.

Dans son essai Qu'est-ce qu'une traduction relevante, Derrida (2005), joue sur le mot «relevante». Il montre comment ce terme "porte en son corps une opération de traduction en cours» (p. 8). Il y a dans ce mot la mémoire de la notion anglaise «relevant», qui soulève la question de la pertinence d'une traduction. Mais l'expression féminine «relevante» issue du verbe «relever» en français nous fait entrer dans un autre registre de sens. Il s'agit d'un participe présent, d'une activité en train de se faire, à l'envers de l'adjectif anglais «relevant» qui qualifie une action déjà faite. «Relever» en français peut signifier reconstruire, rétablir, remonter, rehausser, assaisonner, pimenter, etc. On relève un plat, on relève ses manches, on relève un défi. Quelque chose de relevant, c'est donc aussi quelque chose qui élève, stimule, vivifie. 


\section{Quel type d'interface de traduction valoriser?}

Si la recherche et les pratiques ne sont pas dans un rapport dichotomique et que la traduction n'est pas un processus de transfert des connaissances, alors les médias traditionnels (i.e. les revues professionnelles, les formations initiales, les conférences professionnelles) devraient aussi être repensés. Mais comment le savoir peut-il se transmettre à l'extérieur de ces médias classiques de traduction? L'acception de la traduction retenue ici invite un autre rapport à la recherche, un rapport plus intime. Le savoir n'est pas quelque chose que l'on transfere ou transmet, mais quelque chose que l'on développe et s'approprie.

La figure du rhizome (Deleuze \& Guattari, 1980) est représentative d'une épistémologie de la traduction. Elle s'oppose à la figure plus classique de l'arbre, qui possède des racines, un tronc et des feuilles. L'arbre croît dans une seule direction et la santé de ses feuilles dépend de la vigueur de ses racines. Comme le savoir traditionnel, il se développe suivant un ordre hiérarchique, alors que le rhizome croît sous la terre, à l'abri des regards, dans des directions multiples et imprévisibles, comme la traduction. Le rhizome n'a pas de centre, de sens unidirectionnel et chaque élément peut donc en influencer un autre. Selon cette structure, la recherche peut se développer dans une multiplicité de directions, donner naissance à une infinité de nouvelles pratiques, de nouvelles idées qui, à leur tour, peuvent en générer d'autres. Ce sont les praticiens qui deviennent traducteurs, ils participent à donner sens à leur pratique.

Dans ce cadre, un espace de traduction est un lieu de partage et d'appropriation contrôlé par les usagers eux-mêmes. Le développement croissant de blogues professionnels, de cafés pédagogiques ou toute autre forme de réseaux informels sur internet représente bien ce type d'espace où le savoir se développe dans des directions multiples, où les praticiens participent et s'engagent activement dans ce développement. Il est intéressant de noter qu'on parle assez sou-vent d'interfaces de traduction, un terme largement teinté par le domaine informatique: on parle d'interfaces java, web, numérique, etc. Ces espaces sont des lieux de transmission, mais leur but n'est pas de diffuser efficacement des informations prétablies: leur but est de rendre les échan-ges et les interactions le plus fertile et efficace possible.

Nous sommes encore en train d'explorer les possibilités que les nouvelles technologies offrent. Leur fertilité est évidente car elles permettent aux acteurs de s'engager directement dans leur propre développement, de se remettre en question, de créer, d'innover, et de partager les idées qui les animent. Ces interfaces offrent un lieu de rencontre ouvert à la "conversation", dans le sens entendu par Cavell, c'est-à-dire un espace de conversation libre (non hiérarchique) et ininterrompue que les acteurs peuvent s'approprier et employer aux fins qui les concernent. Les conversations peuvent prendre des directions multiples et parfois inattendues, les personnes y sont engagées, elles ne sont plus seulement des utilisatrices passives de connaissances produites par d'autres. Ces espaces permettent donc une véritable réappropriation du métier par ceux qui le pratiquent. 


\section{Conclusion}

En s'inspirant des travaux issus de la philosophie de l'éducation et en adoptant une perspective empruntée à la philosophie du langage, cette contribution a tenté de se distancier des acceptions habituellement retenues pour discuter de la traduction de la recherche en pratiques en éducation. Grâce à ce procédé de défamiliarisation, l'idée de "traduire la recherche en pratiques» prend un sens suffisamment différent pour sortir de la dualité recherche/pratique: la recherche devient un acte de transformation et d'engagement inhérent à la pratique. Elle peut aider les praticiens à trouver l'énergie, l'inspiration, la volonté de se mettre au travail. Dans ce cadre, les praticiens assument eux-mêmes le travail de traduction, d'où la pertinence d'interfaces informelles qui leur permettent de diriger leur pensée et leurs efforts dans des directions multiples qui ne connaissent pas de limites. Certainement, ces idées ne sont pas entièrement nouvelles. L'idée d'engagement des praticiens dans leur propre développement professionnel, l'idée de développement de communautés de pratiques et d'interfaces de clavardage existent déjà. Cet essai peut donc peut-être se lire comme un manifeste en faveur de telles pratiques au nom de la traduction.

\section{Notes}

1 Oustinoff (2013) reprend ici les propos de Schleiermacher dans «Différentes méthodes de traduire», (Conférence lue le 24 juin 1813 à l'Académie Royale Des Sciences de Berlin.), traduit par Antoine Berman. (1999). Paris: Éd. du Seuil, pp. 31-57.

2 On peut trouver d'autres exemples de cette conception du langage et de la traduction dans les travaux de Jacques Derrida, Mikhail Bakthine, Henri Meschonnic.

3 Le document du MELS (2009) rend compte d'au moins cinq formes que l'adaptation de la recherche peut prendre: l'adaptation simple présente les résultats de recherche dans un langage simple non technique; l'adaptation didactique fournit des exemples ou des démonstrations sur la manière d'utiliser les résultats de recherche; l'adaptation tactique présente des documents ou des produits sous des formes attrayantes (p. ex. l'attention accordée à la conception graphique, aux couleurs, à l'humour, à la présentation, etc.); l'adaptation thématique prépare des rapports sur des sujets particuliers; l'adaptation dialogique discute des répercussions des résultats de recherche avec les utilisateurs.

\section{Bibliographie}

Austin, J. L. (1975). How to do things with words.( Second edition). Oxford: Harvard University Press.

Benjamin, W. (2000/1923). The Task of the Translator. In Lawrence Venuti (Éd.). The Translation Studies Reader. London: Routledge.

Berghdal, L. (2009). Lost in translation: on the untranslatable and its ethical implications for religious pluralism. Journal of Philosophy of Education, 42, (4), 31-44.

Bissonnette, S., Richard, M. \& Clermont Gauthier, C. (2005). Échec scolaire et réforme éducative, quand les solutions proposées deviennent la source du problème. Québec: Presses de l'Université Laval.

Cavell, S. (2004). Cities of words: pedagogical letters on the moral life. Cambridge, Mass.: The Belknap Press of Harvard University Press. 
Conseil supérieur de l'éducation. (2006). Le dialogue entre la recherche et la pratique en éducation: une clé pour la réussite. Gouvernement du Québec.

Deleuze, G. \& Guattari, F. (1980). Mille Plateaux. Paris: Éditions de Minuit.

Derrida, J. (2005). Qu'est-ce qu'une traduction relevante. Paris: L'Herne.

Derrida, J. (1979). L'écriture et la différence. Paris: Éditions du Seuil.

Derrida, J. (1972). La dissémination. Paris: Éditions du Seuil.

Derrida, J. (1972b). Signature, événement, contexte. Marges - de la philosophie. Paris: Éditions de Minuit.

Dobson, S. (2012). The pedagogue as translator in the classroom. Journal of Philosophy of Education, 46, (2), 271-286.

Hammersley, M. \& Gomm, R. (2002).Research and practice, two worlds for ever at odds? In M. Hammersley (Éd.). Educational research, policymaking and practice (pp. 59-83). London: Paul Chapman Publishing.

Harris, S. (2009). Translation, internationalisation and the university. London Review of Education, 7, (3), 223-33.

Higgins, C. \& et Burbules, N. C. (2011). Teaching and translation. PES (Philosophy of Education Society) annual conference. Urbana, Illinois.

Hofstetter, R., Schneuwly, B. \& Lussi, V. (2005). Analyse critique des approches théoriques et historiques portant sur les interactions entre sciences de l'éducation et professionnalisation des enseignants au 20e siècle: enjeux de savoirs et de pouvoirs. $9^{e}$ rencontre $d u$ Réseau Éducation et Formation (REF), Montpellier.

Jbilou, J. (2010). Adaptation des résultats de recherche. Concepts et mesures. Thèse de doctorat présentée à la Faculté des études supérieures de l'Université Laval, Québec.

Labaree, D. F. (2003). «The peculiar problems of preparing educational researchers.» Educational Researcher, 32, (1), 13-22.

Lantheaume, Françoise. (2007). L'activité enseignante entre perception et réel: ruses, petits bonheurs, souffrance. Éducation et Sociétés, 19, 1, 67-83.

Meschonnic H. (1973). Pour la poétique II. Épistémologie de l'écriture. Poétique de la traduction. Paris: Gallimard.

Oustinoff, M. (2003). La traduction. Paris: Puf.

Québec. (2009). (Sous la direction de Réjean Landry). La recherche, comment s'y retrouver? Revue systématique des écrits sur le transfert des connaissances en éducation. Texte consulté en ligne le 2 octobre 2013 à l'adresse suivante: http://www.mels.gouv.qc.ca/sections/publications/index.asp?page $=$ fiche $\&$ id $=803$

Québec. (2006). Programme de formation de l'école québécoise. Ministère de l'Éducation, des Loisirs et du Sport du Québec.

Ruitenberg, C. (2009). Distance and defamiliarisation: translation as philosophical method. Journal of Philosophy of Education, 43, (3), 421-435.

Ost, F. (2009). Traduire. Défense et illustration du multilinguisme. Paris: Fayard.

Saito, N. (2009). Ourselves in translation: Stanley Cavell and philosophy as autobiography. Journal of Philosophy of Education, 43, (2), 253-267.

Schön, D. A. (1994). Le praticien réflexif: à la recherche du savoir caché dans l'agir professionnel. Montréal: Éditions Logiques.

Standish, P. (2005). Towards an economy of higher education. Critical Quarterly, 47, (1), 1-2.

Standish, P. (2011). Social justice and the Occident. Tenth East-West Philosophers' Conference. Honolulu, Hawaii.

Standish, P. (2010). One language, one world: the common measure of education. Philosophy of Education Yearbook.

Mots-clés: Traduction, professionnalisation des enseignants, transfert des connaissances en éducation, changement en éducation. 


\section{Forschungs-Praxis-Transfer: Welche Umwandlung muss vorgenommen, welche Verpflichtung muss eingegangen werden?}

\section{Zusammenfassung}

Ausgehend von Arbeiten aus dem Bereich der Philosophie der Pädagogik und in der Perspektive der Philosophie der Sprache versucht dieser Essay sich von gemeinhin akzeptierten Konzeptionen zu distanzieren, um die Übersetzung der Forschung in die pädagogische Praxis zu diskutieren. Nach einer kurzen Präsentation von Sprache als Übersetzung wird vorgeschlagen, die Übersetzung als Analysemodell einzusetzen, und zwar unter Einbezug der Arbeiten von Paul Standish zur sozialen Gerechtigkeit. Auf der Basis dieses Modells untersucht der Artikel eine wichtige Debatte in der Erziehungswissenschaft: die Frage nach der Beziehung zwischen Forschung und Praxis. Anschliessend werden zwei weitere durch das Modell zur Sprache gebrachte Themenkreise untersucht: (i) die Frage nach den Kriterien, die für die Evaluation der Qualität und Relevanz von Forschung zum Einsatz kommen, sowie (ii) die Frage nach den Übersetzungsschnittstellen. Damit sollen einige Auswirkungen für den Unterricht, für die Forschung in der Pädagogik sowie für die Ausbildung der Lehrpersonen aufgezeigt werden.

Schlagworte: Übersetzung, Professionalisierung des Lehrberufs, Wissenstransfer in Bildung und Erziehung, Umbruch in der Erziehung.

\section{Tradurre la ricerca nella pratica: verso un atto di trasformazione e di impegno}

\section{Riassunto}

Traendo ispirazione dai lavori sul tema della filosofia dell'educazione e adottando una prospettiva improntata alla filosofia del linguaggio, questo saggio rappresenta un tentativo di distanziarsi dalle concezioni abituali per lanciare una discussione sulla traduzione delle ricerca in pratiche dell'educazione. Dopo una breve presentazione del linguaggio come traduzione, propone di prendere la traduzione come modello per l'analisi del «transfer di conoscenze» riallacciandosi ai lavori di Paul Standish sulla giustizia sociale. Basandosi su tale modello, esamina un dibattito importante delle scienze dell'educazione, ovvero il rapporto tra la ricerca e la pratica. Infine, l'articolo presenta due interrogativi sollevati da questo modello: in primo luogo la questione dei criteri che consentono di giudicare la qualità e la pertinenza della ricerca e, in secondo luogo, l'aspetto delle interfacce che, rispettivamente, separano o uniscono la ricerca e la pratica in tale contesto. Ciò comporta delle implicazioni per quanto riguarda l'insegnamento, la ricerca in educazione e la formazione dei docenti. 
Parole chiave: Traduzione, professionalizzazione dei docenti, transfer delle conoscenze in educazione, cambiamento nell'educazione.

\section{Translating research into practice: towards an act of transformation and commitment}

\section{Summary}

Drawing on works in philosophy of education and adopting a perspective borrowed from the philosophy of language, this paper attempts to distance itself from commonly accepted conceptions in order to discuss the translation of research into educational practice. Following a brief presentation of translation as an act of transformation and commitment, it assesses the fertility of this model drawing on Paul Standish's work on social justice. Based on this model, this paper examines an important debate in educational research: the relationship between research and practice. It adresses two issues raised by this model, the question of the criteria used in order to judge the quality and relevance of research, and the question of translation interfaces. This has certain implications for teaching, educational research and teacher training.

Keywords: Translation, professionalization of teaching; knowledge transfer in education, educational change. 